\title{
Micropulse diode laser trabeculoplasty (MDLT): A phase II clinical study with 12 months follow-up
}

\author{
Antonio Maria Fea \\ Alex Bosone \\ Teresa Rolle \\ Beatrice Brogliatti \\ Federico Maria Grignolo \\ Istituto di Fisiopatologia Clinica, \\ Clinica Oculistica dell' Università \\ di Torino, Torino, Italy
}

\begin{abstract}
Objective: This pilot study evaluates the pressure lowering potential of subthreshold micropulse diode laser trabeculoplasty (MDLT) for a clinically meaningful duration in patients with medically uncontrolled open angle glaucoma (OAG).

Design: prospective interventional case series.

Participants: Thirty-two eyes of 20 consecutive patients with uncontrolled OAG (12 bilateral and 8 unilateral).

Methods: Confluent subthreshold laser applications over the inferior $180^{\circ}$ of the anterior TM using an $810 \mathrm{~nm}$ diode laser in a micropulse operating mode. The intraocular pressure (IOP) was measured at baseline and at 1 hour, 1 day, 1 week, 3, 6, 9, and 12 months post-treatment. Flare was measured with a Kowa FM 500 flare-meter at baseline and at 3 hours, 1 day, 1 week, and 12 months post-treatment. After treatment, the patients were maintained on their pre-treatment drug regimen.
\end{abstract}

Main outcome measures: Criteria for treatment response were IOP reduction $\geq 3 \mathrm{~mm} \mathrm{Hg}$ and IOP $\leq 21 \mathrm{~mm} \mathrm{Hg}$ within the first week after MDLT. Eyes not complying to the above criteria during the follow-up were considered treatment failure. Mean IOP change and percentage of IOP reduction during the follow-up were calculated.

Results: One eye was analyzed for bilateral patients. A total of 20 eyes were thus included. Four eyes (20\%) did not respond to treatment during the first week. One additional eye failed at the 6 month visit. The treatment was successful in 15 eyes (75\%) at 12 months. The IOP was significantly lower throughout follow-up $(\mathrm{p}<0.01)$. At 12 months, the mean percentage of IOP reduction in the 15 respondent eyes was $22.1 \%$ and 12 eyes $(60 \%)$ had IOP reduction higher than $20 \%$. During the first two postoperative days, one eye with pigmentary glaucoma experienced a significant increase of flare associated with an IOP spike $(34 \mathrm{~mm} \mathrm{Hg}$ ) that was controlled with systemic drugs; afterwards it qualified as a respondent and completed the study. No increase of flare was found in any other patient. No peripheral anterior synechiae formed.

Conclusions: In this case series, MDLT was effective in reducing IOP in $75 \%$ of medically insufficiently controlled OAG eyes without significant complications. This justifies randomized clinical studies to compare MDLT with current IOP lowering strategies.

Keywords: open angle glaucoma, trabeculoplasty, diode laser, micropulse, subthreshold

\section{Introduction}

Following the publication of Wise and Witter's pilot study on 40 eyes treated with argon laser trabeculoplasty (ALT) in 1979 (Wise and Witter 1979), ALT became a commonly performed procedure for the treatment of primary open-angle glaucoma (POAG), exfoliation syndrome glaucoma, pigmentary glaucoma, and normal-tension glaucoma.

On the basis of the evidence from the Glaucoma Laser Trial Follow-up Study (GLTFS) (GLTRG 1995), the investigators reported that ALT is superior to medications as first-line therapy in newly diagnosed POAG. Nevertheless, ALT has not become a primary treatment of choice; it has mainly been used as an adjunctive 
therapy, reserved for patients with poorly controlled intraocular pressure (IOP) under maximum tolerated medical therapy. While the efficacy of ALT in reducing IOP has been documented, side effects are frequently encountered, including IOP spikes in the early post-ALT period, local scarring of the trabecular meshwork, onset of peripheral anterior synechiae, and limited IOP response to repeated ALT (Wise and Witter 1979; Wilensky and Weinreb 1983; Traverso et al 1984; Feldman et al 1991).

In 1995, Latina and Park (1995) introduced selective laser trabeculoplasty (SLT) and noted reduced laser collateral damage and fewer ALT side-effects. SLT utilizes a single 3 ns duration pulse of a $532 \mathrm{~nm}$ laser with large $400 \mu \mathrm{m}$ spot to selectively destroy pigmented TM cells with no damage to adjacent nonabsorbing cells in accordance with the principles of selective photothermolysis (Anderson and Parrish 1983). In several studies, SLT has produced IOP-lowering effects comparable with ALT (Damji et al 1999; Reno et al 1999; Juzych et al 2004) with a lower, but still considerable, post-operative inflammation and occurrence of pressure spikes, especially in eyes with heavily pigmented TM (Martinez-de-la-Casa et al 2004; Harasimowycz et al 2005).

Trabeculoplasty with subvisible applications (subthreshold) of repetitive short diode laser pulses, named micropulse diode laser trabeculoplasty (MDLT) to differentiate it from the conventional continuous wave $(\mathrm{CW})$ diode laser trabeculoplasty (DLT) (McHugh et al 1990; Brancato et al 1991), was tested in a short-term prospective controlled pilot study in which patients with uncontrolled OAG were randomized to either MDLT or ALT (Ingvoldstad et al 2005). At the 3-month follow-up visit, the IOP reduction was statistically significant and comparable in both study arms, but, with MDLT, intraoperative pain, postoperative inflammation, and cell/flare reaction were negligible and significantly lower than with ALT.

The objective of the present study was to evaluate the pressure lowering potential of MDLT in eyes with uncontrolled OAG over a longer and clinically meaningful followup period of 12 months.

\section{Materials and methods Study design}

Prospective pilot study of consecutive patients with uncontrolled OAG on maximal tolerated medical therapy.

\section{Eligibility and exclusion criteria}

Consecutive patients were eligible for the study if they met the following entry criteria: 1) an average IOP greater than $22 \mathrm{~mm}$
$\mathrm{Hg}$ at three pre-treatment visits in separate days within two weeks; and 2) a clinical history in our clinic of at least two years without clinically significant IOP variations that could suggest a good compliance to medical treatment. Patients were excluded if they presented with: 1) evidence of glaucoma other than $\mathrm{OAG}$ (angle-closure, neovascular); 2) any previous glaucoma surgery (including ALT and laser iridotomy); 3) ocular conditions which may require ocular surgery; and 4) severe visual field (Humphrey) defect in both eyes according to the Hodapp classification (Hodapp et al 1993). Ethics committee approval was obtained. All eligible patients entered in the study signed an informed consent form acknowledging their understanding of the goals, procedures, and risks of the study.

\section{Subjects}

Twenty out of twenty-eight consecutive patients gave their consent and entered the study with a total of 32 eligible eyes ( 8 unilateral and 12 bilateral). Baseline data for each patient included full ocular and medical history, slit lamp biomicroscopy with gonioscopy, flare determination using the Kowa FM 500 flare-meter, pachimetry, mydriatic funduscopy, and visual field assessment (Humphrey 24-2). At least three IOP measurements were taken with the Goldmann applanation tonometer on separate days, between 8.30 and 9.30 a.m. within two weeks before the treatment. IOP measurements were not adjusted for central corneal thickness. The median of the measurements was taken as the baseline IOP.

\section{Laser technique}

We did MDLT with the IRIS Medical OcuLight SLx 810 diode laser system (IRIDEX Corporation, Mountain View, CA, USA), a commercially available laser device, FDA-cleared for various indications including retinal photocoagulation, laser trabeculoplasty, transscleral cyclophotocoagulation, and other diode laser ocular treatments. The micropulse laser settings, largely derived from the parameters clinically tested in a prospective, randomized pilot study (Ingvoldstad et al 2005) were as follows: $200 \mu \mathrm{m}$ spot size diameter, $2 \mathrm{~W}$ power $\left(6.4 \mathrm{KW} / \mathrm{cm}^{2}\right.$ irradiance), and $200 \mathrm{~ms}$ duration with $15 \%$ duty cycle. With these settings, a train of 100 laser pulses with $300 \mu \mathrm{s}$ "ON" time, each separated by $1700 \mu \mathrm{s}$ "OFF" time, is delivered with each $200 \mathrm{~ms}$ application. Patients received a single MDLT treatment and no retreatment was permitted.

All MDLT procedures were performed by the first author (AMF). After a drop of topical anesthetic (Oxybuprocaine $0.4 \%$ with methylcellulose), the patient was seated at the slit lamp and a laser antireflective coated Goldmann three-mirror 
lens (Ocular Instruments, Bellevue, WA, USA) was placed on the eye to be treated. The laser was carefully focused on the anterior trabecular meshwork and confluent applications were administered over the inferior $180^{\circ}$. Since no visible laserinduced tissue change endpoint is produced at the TM with the above laser parameters, and due to eye movements during the treatment, the placement of invisible confluent applications over the inferior 180 degrees relied on the surgeon's judgment and skill, resulting in a variable number of confluent and even overlapping spots. The total number of laser applications delivered to each eye was recorded after each treatment.

\section{Post-operative follow-up}

Immediately after the treatment, indomethacin $0.1 \%$ eye drops were administered and continued three more times during the first day. Nonsteroidal anti-inflammatory eye drops were chosen instead of steroidals due to the potential confounding effect of steroidal eye drops in glaucomatous responders. IOP was assessed in all patients 3 hours after the laser treatment and further monitored in case of IOP raised $>3 \mathrm{~mm} \mathrm{Hg}$. Systemic acetazolamide (Diamox $250 \mathrm{mg}$ ) was the treatment of choice for IOP spikes in order to minimize confounding long term IOP-lowering effects hiding the effects of the laser treatment. As soon as IOP normalized, acetazolamide was discontinued. All IOP measurements made during the treatment with acetazolamide were excluded from the analysis. Patients were evaluated at 1 day, 1 week, 1 month, 3 months, 6 months, 9 months, and 12 months. At each visit, anterior segment examination, best corrected visual acuity and IOP measurements were performed. Flare was measured at baseline, at 3 hours, 1 day, 1 week, and 1 year after the treatment. At the one year examination, gonioscopy and a visual field test were performed. All the post-operative visits were performed by observers unaware of the treatment and of the patients' clinical history (AB and TR). IOP was always measured between 8.30 and 9.30 am to minimize the effects of diurnal variations. Preoperative medications were maintained unchanged throughout the course of the study. During the first post-op week, patients who did not achieve an IOP reduction $>3 \mathrm{~mm} \mathrm{Hg}$ or whose IOP was $>21 \mathrm{~mm} \mathrm{Hg}$ were considered nonresponders and excluded from further study follow-up to allow changes in their therapy, as deemed appropriate by the physician. Furthermore, any eye with IOP greater than $21 \mathrm{~mm} \mathrm{Hg}$ or that did not have an IOP reduction higher than $3 \mathrm{~mm} \mathrm{Hg}$ at any time point was considered a failure and the patient was excluded from further study follow-up to allow appropriate therapy changes.

\section{Measures of outcome}

Eyes were censored from further analysis upon nonresponse or failure. The mean IOP at each visit was compared using twotailed paired t test. The percentage of IOP reduction and the mean IOP reduction throughout the visits was calculated.

\section{Results}

The baseline demographic characteristics are listed in Table 1.

In all, 32 eyes of 20 patients ( 8 unilateral and 12 bilateral) were enrolled in the study. Since both eyes had similar reaction in bilateral patients, one eye per patient was analyzed. Overall 20 eyes were then included in the study. At the first week follow-up visit 4 eyes (20\%) were found not respondent to treatment: 3 eyes $(15 \%)$ did not achieve an IOP reduction $>3 \mathrm{~mm} \mathrm{Hg}$ and 1 eye $(5 \%)$ retained an IOP $>22 \mathrm{~mm} \mathrm{Hg}$. At the sixth month follow-up visit, one eye $(5 \%)$ failed because the IOP reduction was less than 3 $\mathrm{mm} \mathrm{Hg}$ from baseline.

The mean IOP had a prompt and significant decrease $(\mathrm{F}=45.91 ; \mathrm{p}<0.01)$ that was maintained during the 12 month follow-up as shown in Figure 1 and reported in Table 2 .

Figure 2 shows the distribution of the percentage of eyes with IOP reduction $<20 \%$, between $20 \%-29 \%$ and $\geq 30 \%$ for the 15 eyes that fulfilled our success criteria through the entire followup. Ten of the 15 eyes $(66.67 \%$ ) had an IOP reduction $\geq 20 \%$.

Table I Baseline demographic characteristics

\begin{tabular}{|c|c|}
\hline Age (years) & $61.7 \pm 6.3$ \\
\hline Sex & $8 \mathrm{M} ; 12 \mathrm{~F}$ \\
\hline \multirow{12}{*}{$\begin{array}{l}\text { Number of glaucoma } \\
\text { medications }\end{array}$} & I drop: 4 eyes (prostaglandins) \\
\hline & 2 drops: 18 eyes \\
\hline & 10 eyes beta-blockers \\
\hline & and prostaglandins \\
\hline & 2 eyes $\mathrm{CAl}$ and \\
\hline & prostaglandins \\
\hline & 6 eyes beta-blockers \\
\hline & and $\mathrm{CAl}$ \\
\hline & 3 drops: 10 eyes \\
\hline & 8 eyes beta-blockers, \\
\hline & $\mathrm{CAl}$ and prostaglandins \\
\hline & $\begin{array}{l}2 \text { eyes beta-blockers, } \alpha_{2} \text {-agonists, } \\
\text { and prostaglandins }\end{array}$ \\
\hline Mean IOP (mm Hg) & $24.7 \pm 2.0$ \\
\hline $\begin{array}{l}\text { Central corneal } \\
\text { thickness }(\mu \mathrm{m})\end{array}$ & $538.5 \pm 11.53$ \\
\hline Mean Defect $(\mathrm{dB})$ & $-7.4 \pm 0.85$ \\
\hline
\end{tabular}

Abbreviations: CAl, carbonic anhydrase inhibitors; IOP, intraocular pressure. 


\section{IOP vs time}

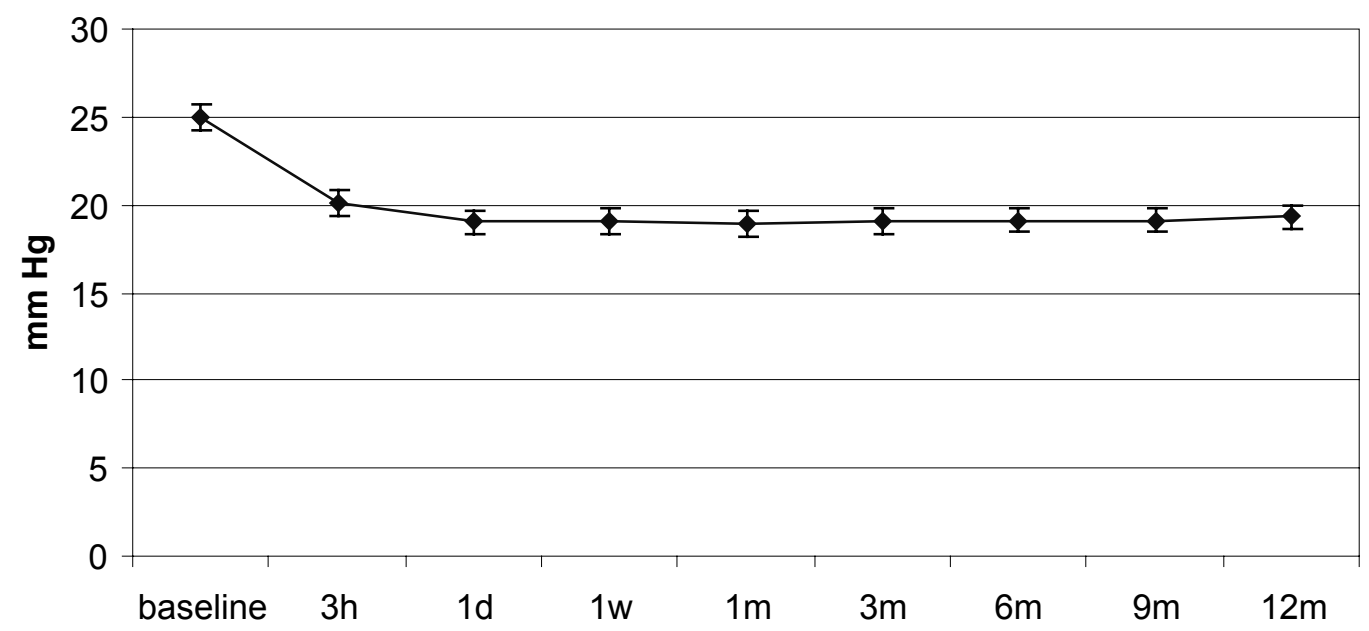

Figure I Mean intraocular pressure (IOP) at various time points up to 12 months (mean $\pm \mathrm{SE}$ ).

The distribution, skewed to the left, indicates a small increase with time of eyes with IOP reduction $<20 \%$ from baseline.

The mean flare value at the Kowa FM 500 flare-meter was not significantly increased from the preoperative $10.12 \pm 6.7$ fotons/msec throughout all the follow-up period $(10.4 \pm 5.3$ at the 12 th month).

A marked increase in flare occurred immediately after MDLT in the eye of a unilaterally treated patient with pigmentary glaucoma. His IOP rose to $34 \mathrm{~mm} \mathrm{Hg}$ and was controlled with acetazolamide (Diamox $250 \mathrm{mg}$ ) for two days. On the third day, the IOP normalized and Diamox was discontinued. The IOP measurements taken during the two days on acetazolamide are not included in the analysis. No other patient had IOP or flare spikes. The MDLT treatment was generally well tolerated with no pain and no annoying visible laser flashes. The total number of laser applications delivered at each eye over the inferior $180^{\circ}$ ranged from a minimum of 70 to a maximum of 84 (mean $74.25 \pm 3.8$ ). Burning sensation was reported in two eyes (one patient) and heat sensations in four eyes (three patients). Treatments were completed with no complications. At the one year follow-up no patient had peripheral anterior synechiae. Visual acuity and visual field were unchanged in all eyes.

\section{Discussion}

The objective of this prospective case series study was to evaluate the pressure lowering potential of MDLT in eyes with uncontrolled OAG over a clinically meaningful period. This is the first published report on MDLT with a 12-month follow-up.

A previous controlled pilot study was presented by Ingvoldstad and colleagues (2005) at the 2005 ARVO meeting. They compared the response to ALT and MDLT during a three-month follow-up period. These investigators reported similar, statistically significant IOP reductions with the two techniques: $18.9 \%$ with ALT and $18.3 \%$ with MDLT.

We considered successful eyes only in our study because we operated on the eyes that failed. It was then impossible to compare the results obtained with surgery with those obtained by the trabeculoplasty method.

Table 2 Mean intraocular pressure (IOP) with standard deviation and mean IOP reduction from baseline at various time points up to 12 months. The results of the paired t-test comparing the mean IOP at each time point to baseline are also shown

\begin{tabular}{|c|c|c|c|c|c|c|c|c|c|}
\hline & Baseline & 3 Hours & I Day & I Week & I Month & 3 Months & 6 Months & 9 Months & 12 Months \\
\hline & $N=20$ & $N=20$ & $N=19$ & $N=16$ & $N=16$ & $N=16$ & $N=16$ & $N=15$ & $N=15$ \\
\hline Mean IOP (mm Hg) & 25 & 20.1 & 19.6 & 18.7 & 18.9 & 19.2 & 19.2 & 19.3 & 19.5 \\
\hline Standard deviation & 2.1 & 2,4 & 1,2 & $\mathrm{I}, 8$ & 1.7 & 1.3 & 1.4 & 1.7 & 1.7 \\
\hline Range & $23-30$ & $16-34$ & $17-26$ & $|6-2|$ & $|6-2|$ & $|6-2|$ & $|7-2|$ & $|6-2|$ & $|6-2|$ \\
\hline $\mathrm{T}$ & & 6.8 & 10.9 & 8.9 & 9.4 & 9.8 & 9.6 & 8.9 & 8.6 \\
\hline $\mathrm{P}$ & & $<0,001$ & $<0,001$ & $<0,001$ & $<0,001$ & $<0,001$ & $<0,001$ & $<0,001$ & $<0,001$ \\
\hline $\begin{array}{l}\text { Mean IOP } \\
\text { reduction \% }\end{array}$ & & $20.8 \%$ & $20.9 \%$ & $21.0 \%$ & $23.8 \%$ & $22.5 \%$ & $22.2 \%$ & $22.0 \%$ & $21.3 \%$ \\
\hline
\end{tabular}




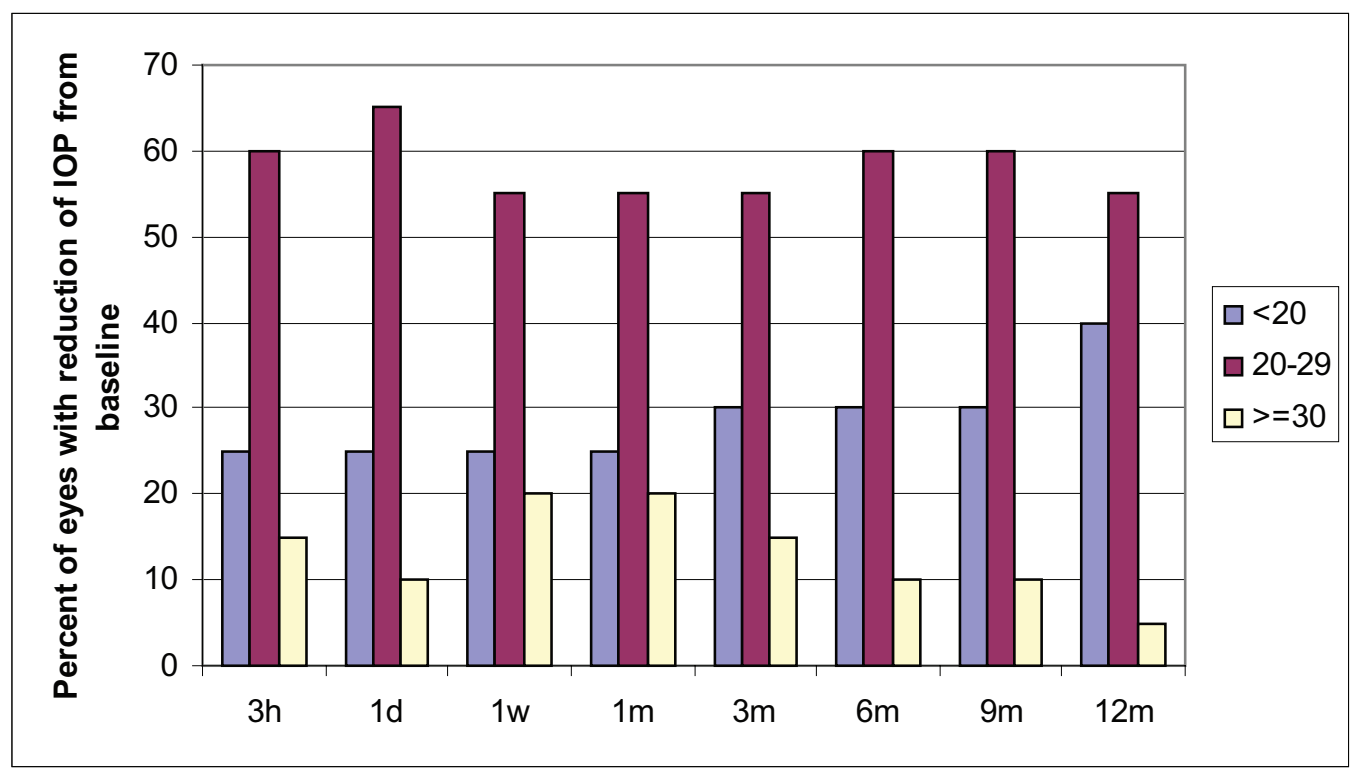

Figure 2 Distribution of the percentage of eyes with intraocular pressure (IOP) reduction from baseline $<20 \%$, between $20 \%-30 \%$.

It is possible that we may have prematurely excluded the six nonresponders' eyes and that some of them would have had late response to MDLT after the first week. Positive late response to laser trabeculoplasty has been reported for ALT (Weireb et al 1983) and SLT (Latina and de Leon 2005), but a similar delayed effect could not be identified in our study due to giving modified therapy for these patients.

Due to our small number of eyes, it is not possible to identify individual patient characteristics and laser factors (ie, parameters for individual pigmentation, appropriate focussing, etc.) that might have influenced either a nonresponse or a slower late response.

The micropulse laser energy delivered to the TM with our settings did not produce clinically visible morphologic changes at any time point. The lack of visible tissue changes constitutes a clinical challenge because, in the absence of a visible endpoint, the treatment relies on the surgeon's skill; this could introduce variability. No acute histopathological changes and no damage to the TM beams were found in the histological specimens from an eye enucleated for choroidal melanoma 24 hours after the MDLT treatment with our parameters (Midena 2005).

Except for one of our treated eyes, no postoperative ocular inflammation was detected with the Kowa FM 500 flare-meter or clinical examination. An intraocular pressure spike and a flare reaction occurred in one eye of a patient with pigmentary glaucoma. The IOP elevation was controlled in two days with systemic medication (acetazolamide). Conversely, IOP elevations after SLT in patients with pigmentary dispersion syndrome and with deeply pigmented trabecular meshwork can be a serious adverse event requiring filtering surgery (Latina et al 1998).

The mechanism of action of laser trabeculoplasty is poorly understood, but most recent hypotheses point toward a responsive cellular biochemical cascade (Parshley et al 1996; Bradley et al 2000; Alvarado et al 2005). The threshold of laser-induced cellular effect to activate such a cascade is unknown. The fact that various laser trabeculoplasty techniques - applied with different lasers, endpoints, and protocols - have consistently produced comparable IOPlowering effects supports the hypothesis of a common cellular mechanism. Such a cellular cascade could only occur in viable cells activated by a nonlethal thermal insult, directly applied, as in the case of MDLT, or derived from higher and lethal photothermal elevations produced by the laser in adjacent cells as in the case of ALT and of SLT. This would explain MDLT's pressure lowering effect with subvisible laser applications that produce gentle photothermal effects that may suffice to activate the therapeutic cellular cascade without causing clinically visible damage nor intra- and post-operative side-effects.

The present pilot study has several limitations: a small number of patients, bilateral treatment in some patients, absence of a control group, and the fact that patients were on different preoperative medical treatments. We cannot rule out the possibility that, in some patients, some IOP lowering might have resulted from increased adherence to medical therapy after being entered in the study. This issue was addressed by Latina and colleagues (1998) in a pilot study, in which a very low confounding effect $(0.9-2.1 \mathrm{~mm} \mathrm{Hg})$ sug- 
gested that the amount of IOP reduction from improved compliance is not the predominant reason for response. We have no means to evaluate the impact of a hypothetical improved compliance, but the review of the last two years clinical history available in our clinic for all the patients entered in the study did not reveal clinically significant IOP variations that could flag poor compliance to medical treatment.

Since most of the treatments were bilateral and preoperative medications were maintained during the follow-up, we can exclude any confounding effect due to the contralateral action of drugs.

This preliminary report suggests that some IOP reduction can be achieved in a meaningful proportion of eyes without serious intra-operative and post-operative complications by MDLT. This laser procedure appears to be an alternative to ALT, DLT, or SLT. It is done with a laser device that can also be used for many other ophthalmic applications, thus reducing the economic burden of treatment. Whether a greater IOP reduction could be achieved by extending the treatment to the entire circumference of the angle or through the optimization of laser parameters, for example, by increasing the number and the duty cycle of the micropulses delivered for each application, remain to be investigated. Randomized clinical trials are warranted to compare MDLT with current IOP-lowering laser therapies such as ALT, SLT, or escalated antiglaucoma medications.

\section{Acknowledgements}

We thank Dr. Federica Machetta and Dr. Gianmarco Vizzeri for referring some of the patients included in the study. Regione Piemonte contributed a grant to the research. The authors have no financial interest in this procedure or product. There are no conflicts of interest to report.

\section{References}

Alvarado JA, Alvarado RG, Yeh RF, et al 2005. A new insight into the cellular regulation of aqueous outflow: how trabecular meshwork endothelial cells drive a mechanism that regulates the permeability of Schlemm's canal endothelial cells. Br J Ophthalmol, 89:1500-5.

Anderson RR, Parrish JA. 1983. Selective photothermolysis: precise microsurgery by selective absorption of pulsed radiation. Science, 220:524-7.

Bradley JMB, Anderssohn AM, Colvis CM, et al. 2000. Mediation of laser trabeculoplasty-induced matrix metalloproteinase expression by IL-1 $\beta$ and TNFa. Invest Ophthalmol Vis Sci, 41:422-30.
Brancato R, Carassa R, Trabucchi G, et al. 1991. Diode laser compared with argon laser for Trabeculoplasty. Am J Ophthalmol, 112:50-5.

Damji KF, Shah KC, Rock WJ, et al. 1999. Selective laser trabeculoplasty $\mathrm{v}$ argon laser trabeculoplasty: a prospective randomised clinical trial. Br J Ophthalmol, 83:718-22.

Feldman RM, Katz LJ, Spaeth GL, et al. 1991. Long-term efficacy of repeat argon laser trabeculoplasty. Ophthalmology, 98:1061-5.

[GLTRG] Glaucoma Laser Trial Research Group. 1995. The Glaucoma Laser Trial (GLT) and Glaucoma Laser Trial Follow-up Study: 7. Results. Am J Ophthalmol, 120:718-31.

Harasimowycz PJ, Papamatheakis DG, Latina M, et al. 2005. Selective laser trabeculoplasty (SLT) complicated by intraocular pressure elevation in eyes with heavily pigmented trabecular meshworks. Am J Ophthalmol, 139:1110-13.

Hodapp E, Parrish IIRK, Anderson DR. 1993. Clinical decisions in glaucoma. St Louis, The CV Mosby, pp 84-126.

Ingvoldstad DD, Krishna R, Willoughby L. 2005. Micropulse diode laser trabeculoplasty versus argon laser trabeculoplasty in the treatment of open angle glaucoma [abstract]. Invest Ophthal Vis Sci, 46:123.

Juzych MS, Chopra V, Bantt MR, et al. 2004. Comparison of long-term outcomes of selective laser trabeculoplasty versus argon laser trabeculoplasty in open-angle glaucoma. Ophthalmology, 111:1853-9.

Latina MA, Park C. 1995. Selective targeting of trabecular meshwork cells: in vitro studies of pulsed and $\mathrm{CW}$ laser interactions. Exp Eye Res, 60:359-71.

Latina MA, Sibayan SA, Shin DH, et al. 1998. Q-switched 532-nm Nd:YAG laser trabeculoplasty (selective laser trabeculoplasty): a multicenter, pilot, clinical study. Ophthalmology, 105:2082-8.

Latina MA, de Leon JMS. 2005. Selective laser trabeculoplasty. Ophthalmol Clin North Am, 18:409-41.

Martinez-de-la-Casa JM, Garcia-Feijoo J, Castillo A, et al. 2004. Selective vs argon laser trabeculoplasty: hypertensive efficacy, anterior chamber inflammation, and postoperative pain. Eye, 18:498-502.

McHugh D, Marshall J, Fytche T, et al. 1990. Diode laser trabeculoplasty for primary open-angle glaucoma and ocular hypertension. $\mathrm{Br} J$ Ophthalmol, 74:743-7.

Midena. 2005. Personal communication. AAO 2005, Chicago, IL.

Parshley DE, Bradley JMB, Fisk A, et al. 1996. Laser trabeculoplasty induces stromelysin expression by trabecular juxtacanalicular cells. Invest Ophthalmol Vis Sci, 37:795-804.

Reno JB, Rabban UJ, Shin DH, et al. 1999. A retrospective comparison of selective laser trabeculoplasty versus argon laser trabeculoplasty in chronic open angle glaucoma patients [abstract]. Invest Ophthal Vis Sci, 40:1429.

Traverso CE, Greenidge KC, Spaeth GL. 1984. Formation of peripheral anterior synechiae following argon laser trabeculoplasty. A prospective study to determine relationship to position of laser burns. Arch Ophthalmol, 102:861-3.

Weireb RN, Ruderman J, Juster R, et al. 1983. Influence of the number of laser burns administered on the early results of argon laser trabeculoplasty. Am J Ophthalmol, 95:287-92.

Wilensky JT, Weinreb RN. 1983. Early and late failures of argon laser trabeculoplasty. Arch Ophthalmol, 101:895-7.

Wise JB, Witter SL. 1979. Argon laser therapy for open angle glaucoma: a pilot study. Arch Ophthalmol, 97:319-22. 